\title{
Supermassive recoil velocities for binary black-hole mergers with antialigned spins
}

\author{
José A. González, Mark Hannam, Ulrich Sperhake, Bernd Brügmann, Sascha Husa ${ }^{1}$ \\ ${ }^{1}$ Theoretical Physics Institute, University of Jena, 07743 Jena, Germany
}

\begin{abstract}
Recent calculations of the recoil velocity in binary black hole mergers have found the kick velocity to be of the order of a few hundred $\mathrm{km} / \mathrm{s}$ in the case of non-spinning binaries and about $500 \mathrm{~km} / \mathrm{s}$ in the case of spinning configurations, and have lead to predictions of a maximum kick of up to $1300 \mathrm{~km} / \mathrm{s}$. We test these predictions and demonstrate that kick velocities of at least $2500 \mathrm{~km} / \mathrm{s}$ are possible for equal-mass binaries with anti-aligned spins in the orbital plane. Kicks of that magnitude are likely to have significant repercussions for models of black-hole formation, the population of intergalactic black holes and the structure of host galaxies.
\end{abstract}

PACS numbers: $\quad$ 04.25.Dm, 04.30.Db, 95.30.Sf, 98.80.Jk

Introduction. - A well known phenomenon of general relativity is the recoil or rocket effect due to the emission of anisotropic gravitational radiation [1, 2, 3]. The loss of linear momentum radiated away in the form of gravitational waves imparts a recoil or kick on the remaining system which then moves relative to its original centreof-mass frame. This effect is particularly pronounced for the inspiral and merger of two compact objects and thus may have dramatic consequences for the merger of massive black holes residing at the centers of galaxies when their hosts undergo merger.

Massive black holes with masses $10^{5}$ to $10^{9.5} M_{\odot}$ are not only known to exist at the centre of many galaxies, but also to have a substantial impact on the structure and formation of their host galaxies, as is demonstrated by the correlation of the black-hole mass with the bulge mass, luminosity and velocity dispersion [4, 5, 6, 6]. The merger of galaxies is thus likely to be accompanied by an inspiral of the central black holes. In order to assess the impact of the resulting black-hole recoil on questions such as the bulge structure, the formation history of massive black holes and interstellar and intergalactic populations of black holes, it is vital to have a good understanding of the kick magnitude and, in particular, the maximum possible kick velocities.

The first study of the recoil effect for inspiralling binaries was performed by Fitchett [8] in the framework of non-spinning point particles subject to Newtonian gravity. The resulting recoil calculated using the lowest order multipoles is given in his Eq. (3.21) and predicts kick velocities of the order of $1000 \mathrm{~km} / \mathrm{s}$ that exceed the escape velocities from massive galaxies [9]. The problem was reinvestigated using the particle approximation [10, 11, 12, 13], post-Newtonian methods [14, 15, 16, 17], the Close-Limit Approximation [18, 19, 20] as well as numerical simulations [21, 22]. The picture that emerged from these studies is that the recoil from unequal-mass, non-spinning binaries is unlikely to exceed a few hundred $\mathrm{km} / \mathrm{s}$.

Recent breakthroughs in the numerical simulation of black-hole binaries [23, 24, 25] have enabled investigations of the recoil problem without any restrictive ap- proximations other than the numerical differencing of the Einstein equations. First studies addressed the recoil from non-spinning binaries and confirmed the relatively small magnitude of the kick velocities for mass ratios in the range $1: 1$ to $1: 2$ [26, 27]. In a previous publication [28], we have presented the most comprehensive study of this problem and found a maximum kick of $175.7 \pm 11 \mathrm{~km} / \mathrm{s}$ for a mass ratio $\eta=m_{1} m_{2} /\left(m_{1}+m_{2}\right)^{2}=$ $0.195 \pm 0.005$.

More recent numerical studies have shown, however, that as expected significantly larger kicks are realized if one allows at least one black hole to spin. Simulations of equal-mass binaries with spins orthogonal to the orbital plane predict kick velocities of 475 and $440 \mathrm{~km} / \mathrm{s}$, respectively, in the limit of extreme Kerr black holes [29, 30]. Kicks of tens of $\mathrm{km} / \mathrm{s}$ have been obtained from head-on collisions of spinning black holes 31]. These results are consistent with the effective-one-body postNewtonian (PN) study in 32. In addition, Campanelli, et. al. 33] obtain $v_{\text {kick }}=454 \mathrm{~km} / \mathrm{s}$ in the case of a nonspinning black hole orbiting a spinning counterpart of twice the mass with the spin oriented at $-45^{\circ}$ relative to the orbital plane. They further estimate that an equalmass binary with spins aligned in the orbital plane could produce a maximum kick of around $1300 \mathrm{~km} / \mathrm{s}$ based on a PN calculation by Kidder [15].

In this letter we investigate the scenario suggested by Campanelli, et. al. [15, 33] and find that a kick of $2500 \mathrm{~km} / \mathrm{s}$ is possible - larger than the escape velocity of about $2000 \mathrm{~km} / \mathrm{s}$ of giant elliptical galaxies.

Numerical framework. - The numerical simulations presented in this work were performed independently with the BAM and the LEAN codes. These codes employ the moving-puncture method [23, 24], and are described in detail in [34, 35]. We note, however, the following modifications of the Cactus [36] and Carpet 37] based LEAN code relative to the version presented in [35]: time evolution of the TwoPuncture initial data [38] is performed using the fourth-order accurate Runge-Kutta method, the variable $\phi$ has been replaced by the new variable $\chi=e^{-\phi}$ and the code uses fifth-order prolongation in space. The two codes represent independent 


\begin{tabular}{l|r|r|r|r|r}
\hline \hline Run & $X$ & $P_{y}$ & $m_{i}$ & $S_{x}$ & $v_{\text {kick }}(\mathrm{km} / \mathrm{s})$ \\
\hline MI & \pm 3.257 & \pm 0.133 & 0.363 & \pm 0.2 & $2450 \pm 250$ \\
MII & \pm 4.0 & \pm 0.1125 & 0.287 & \pm 0.2 & 2650 \\
\hline \hline
\end{tabular}

TABLE I: Initial puncture parameters and final kick velocity.

implementations of similar techniques, thereby allowing important cross-validation.

The gravitational waves emitted by the binary are extracted by computing the Newman-Penrose scalar $\Psi_{4}$ over spheres of constant radius at different distances. The notation and equations used for this procedure are explained in detail in section III.A of [34]. In order to estimate the recoil velocity of the system, we compute the total linear momentum radiated during the simulation directly from $\Psi_{4}$ using equation (1) in [28]. The pulse of spurious radiation from the initial data, which would lead to an over-estimation of the final kick by about $5 \%$, is not included in this calculation. We have neglected any recoil that may have accumulated during the earlier inspiral of the black holes; however, for the final kick values we obtain, this introduces an uncertainty of less than $1 \%$ in our results (as obtained from PN estimates in the nonspinning case [16]), which is far less than the uncertainty due to other errors.

We consider configurations with spins of equal size but opposite direction lying in the orbital plane. The initial parameters of Model I (MI) were chosen without particular regard to obtain a quasi-circular configuration. On the other hand, the initial momenta used for Model II (MII) were computed using formula (4.7) from [15] to generate a quasi-circular orbit. The parameters and the values of the kick obtained for the two models are summarized in Table I. The initial configurations, consisting of two equal-mass black holes with spins perpendicular to the orbital angular momentum, are chosen to maximize Eq. (3.31b) in 15] as suggested in [33]. Each black hole has a total mass of $m \sim 0.5$ and we consider spin parameters of the order of $a / m \sim 0.8$, which is below the recent estimates presented in [39].

Results. - We performed evolutions of model MI with the LEAN code using different resolutions and computed the radiated linear momentum at different extraction radii. In Figure 1 we present the recoil speed $v_{z}=-P_{z} / m$ of the final black hole as a function of time for the resolutions $1 / 36,1 / 44$ and $1 / 48$. In this context we note that the $x$ and $y$ components of $v$ are smaller than $1 \mathrm{~km} / \mathrm{s}$, so that the $z$-component is practically identical with the total recoil. The bottom panel of the figure demonstrates fourth-order convergence of the recoil, and we estimate the uncertainty as $43 \mathrm{~km} / \mathrm{s}$ or $1.5 \%$.

The dependence of the recoil velocity on the extraction radius is presented in Figure 2, We find the re- sulting error to be reasonably well approximated by a $1 / r_{\text {ex }}$ falloff and thus obtain uncertainties due to finite extraction radii of $120 \mathrm{~km} / \mathrm{s}$ or $4.5 \%$. Using a conservative error estimate, the magnitude of the final kick is $2450 \pm 250 \mathrm{~km} / \mathrm{s}$.
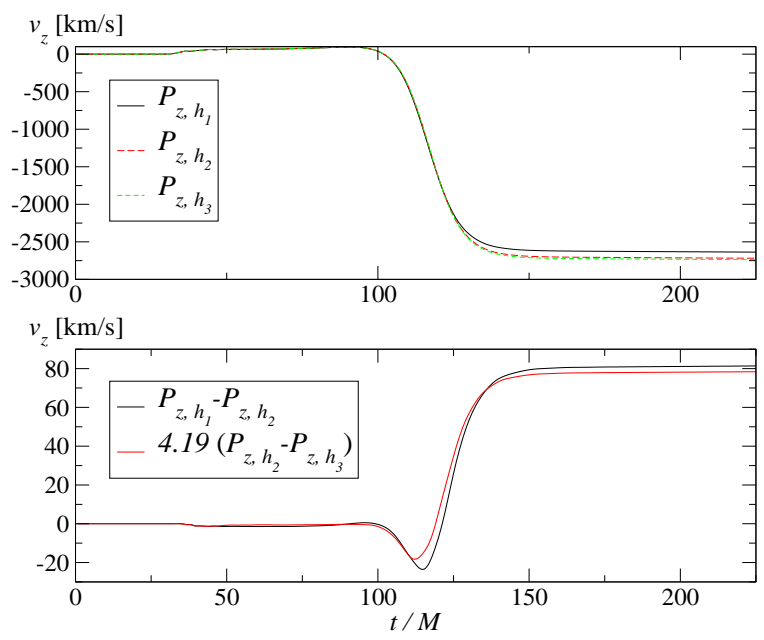

FIG. 1: Upper panel: the $z$-component of the recoil for model MI as a function of time for grid resolutions $h_{1}=1 / 36, h_{2}=$ $1 / 44$ and $h_{3}=1 / 48$. Lower panel: the differences scaled for fourth-order convergence.

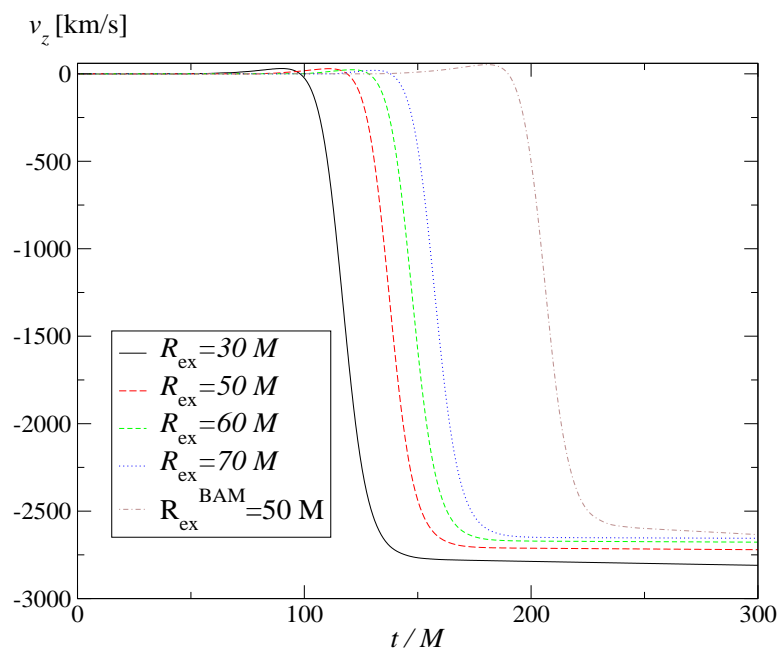

FIG. 2: Recoil for model MI as a function of time for different extraction radii. Extrapolation to $r \rightarrow \infty$ yields a magnitude of $2450 \mathrm{~km} / \mathrm{s}$ with an error estimate of $4.5 \%$. Also shown is the kick from model MII using BAM.

In order to obtain an independent estimate of the kick magnitude for this type of spin-configuration, we evolve model MII using the BAM code. This model starts at larger initial separation and represents a quasi-circular configuration. These simulations thus enable us to assess the uncertainties arising from finite black hole separation 
and deviations from circularity.

The trajectories of the black holes are shown for model MII in Figure 3 and demonstrate how the black holes move out of the initial orbital plane and acquire substantial momentum in the $z$-direction after the merger.

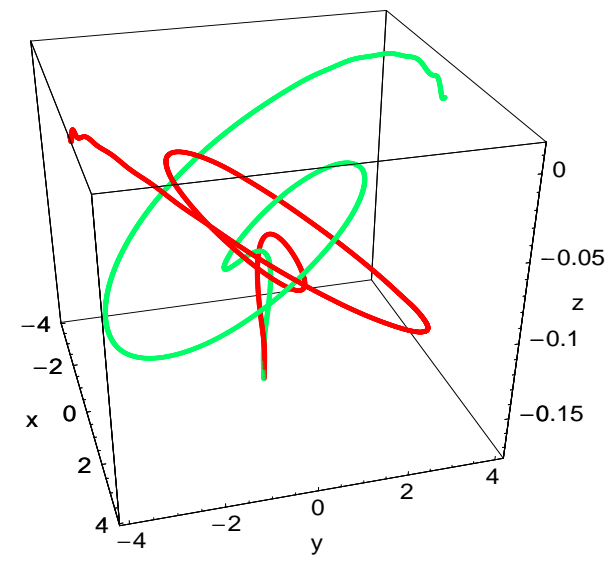

FIG. 3: Coordinate positions of the black-hole punctures for model MII up to $t=180$. The black holes move out of the original plane and after merger the final black hole receives a kick in the negative $z$-direction.

In summary, our simulations predict recoil velocities of $2500 \mathrm{~km} / \mathrm{s}$ with a conservative error estimate of $10 \%$ or $250 \mathrm{~km} / \mathrm{s}$. This value can be compared with the escape velocities from dwarf elliptical and spheroidal galaxies $(\lesssim 300 \mathrm{~km} / \mathrm{s})$ and from giant elliptical galaxies $(\lesssim 2000 \mathrm{~km} / \mathrm{s})$ [40].

Our prediction is larger than any previous numerical result by a factor of five, but other numerical studies considered quite different initial configurations. Since the estimate of $1000 \mathrm{~km} / \mathrm{s}$ [33] for the spins studied here involves a post-Newtonian argument, it is perhaps not surprising that a fully relativistic simulation obtains a different result. This result is very intriguing, but we point out that so far only the two data points presented here are available. It will be important to perform studies at higher numerical resolution, and to study neighboring data sets, for example to vary the initial separation, spin configuration and mass ratio of the black holes.

Astrophysical relevance.- Before we discuss in more detail astrophysical implications of our findings, we emphasize one additional important point: while our results demonstrate that kicks as large as $2,500 \mathrm{~km} / \mathrm{s}$ are possible provided the inspiralling black holes have appropriate spin alignment and magnitude, it does not enable us to make any statements on the probability that these kicks are realized in typical astrophysical merger scenarios. A conclusive assessment of the astrophysical implications therefore requires systematic parameter studies as mentioned in the previous section.

Recoil velocities close to or exceeding the escape ve- locities of the black hole's host give rise to a population of black holes away from the galactic nuclei [41]. In contrast to the non-spinning case, the recoil velocities calculated from our simulations exceed the escape velocities of even massive galaxies and thus imply larger populations of wandering black holes. Observational consequences of wandering black holes have been discussed in detail in Volonteri and Perna [42].

Black-hole recoil has also been found to give rise to a core formation in the central density profile in the host stellar bulge. Boylan-Kolchin et al. [43] find this effect to be most pronounced for recoil velocities just below the escape velocity.

Libeskind, et. al [44] have further found that gravitational recoil manifests itself as a scatter of the relation between black hole and bulge mass. They find this effect to be sensitive to the stability of the disc under galaxy merger. Their simulations indicate a constraint of $v_{\text {kick }} \lesssim 500 \mathrm{~km} / \mathrm{s}$. Merritt, et. al. [45] find constraints of a similar magnitude from narrow emission-line analysis of quasar spectra.

Ejection of black holes affects the rate of binary black hole inspirals in globular clusters and thus the predicted event rates for gravitational wave observatories LIGO, GEO600, TAMA and VIRGO. The latest kick estimates for non-spinning binaries have been taken into account by O'Leary, et. al. [46]. It will be of interest to estimate the impact of spins on such simulations.

The formation history of massive black holes at $z \sim 6$ is often described in the context of hierarchical structure formation via accretion and merger of black holes residing in dark matter halos (see, e.g. [41]). The ejection of black holes from their hosts via gravitational recoil puts constraints on the maximum redshift at which the progenitor seed holes might have started merging and might necessitate accretion growth above the Eddington limit [40, 47] and may also lead to a population of intermediate mass black holes [42]. Black hole mergers would not occur if seed black holes are rare at high redshifts, so that black-hole binaries would not commonly form as a consequence of dark matter halo mergers 48.

Finally, black hole recoil has been suggested to manifest itself directly in observations of off-centre radio-loud active galactic nuclei [48] and off-nuclear ultraluminous X-ray sources in nearby galaxies [49].

Astrophysical studies of the consequences of kick velocities on these scenarios have so far commonly assumed recoil velocities significantly below the values obtained in this work. It will be interesting to see how these consequences are affected by our results. It will also be important to estimate the kick magnitudes in a far wider volume of parameter space.

Note added after preparation of this manuscript: After the first publication on gr-qc of the results reported here, there have been two independent computations of similarly large kicks, first reported in [50] and in an updated 
version of [33]. Since our work is based on the original version of [33], it is relevant to note that the authors have now added new numerical data to their preprint for a configuration showing a kick of $1830 \mathrm{~km} / \mathrm{s}$. They have also modified their argument for the extrapolation from post-Newtonian results (see also [51]) now obtaining an upper limit of $4000 \mathrm{~km} / \mathrm{s}$ [52]. This is consistent with the results we report here and supports the main point we make, namely that kicks much larger than previously expected are indeed realized in numerical simulations with potentially great significance for astrophysics.

This work was supported in part by DFG grant SFB/Transregio 7 "Gravitational Wave Astronomy". We thank the DEISA Consortium (co-funded by the EU, FP6 project 508830), for support within the DEISA Extreme Computing Initiative (www.deisa.org); computations were performed at LRZ Munich and HLRS, Stuttgart. J.G. and U.S. acknowledge support from the ILIAS Sixth Framework Programme.

[1] W. B. Bonnor and M. A. Rotenberg, Proc. R. Soc. Lond. A. 265, 109 (1961).

[2] A. Peres, Phys. Rev. 128, 2471 (1962).

[3] J. D. Bekenstein, Astrophys. J. 183, 657 (1973).

[4] J. Magorrian et al., Astron. J 115, 2285 (1998), astro$\mathrm{ph} / 9708072$.

[5] K. Gebhardt et al., Astrophys. J 539, L13 (2000), astro$\mathrm{ph} / 0006289$.

[6] D. Merritt and L. Ferrarese, MNRAS 320, L30 (2001), astro-ph/0009076.

[7] R. J. McLure and J. S. Dunlop, MNRAS 331, 795 (2002), astro-ph/0108417.

[8] M. J. Fitchett, MNRAS 203, 1049 (1983).

[9] I. H. Redmount and M. J. Rees, Comm. Astrophys. 14, 165 (1989).

[10] M. J. Fitchett and S. Detweiler, MNRAS 211, 933 (1984).

[11] T. Nakamura and M. P. Haugan, Astrophys. J 269, 292 (1983).

[12] M. Favata, S. A. Hughes, and D. E. Holz, Astrophys. J 607, L5 (2004), astro-ph/0408492.

[13] C. O. Lousto and R. H. Price, Phys. Rev. D 69, 087503 (2004), gr-qc/0401045.

[14] A. G. Wiseman, Phys. Rev. D 46, 1517 (1992).

[15] L. E. Kidder, Phys. Rev. D 52, 821 (1995).

[16] L. Blanchet, M. S. S. Qusailah, and C. M. Will, Astrophys. J 635, 508 (2005), astro-ph/0507692.

[17] T. Damour and A. Gopakumar, Phys. Rev. D 73, 124006 (2006), gr-qc/0602117.

[18] Z. Andrade and R. H. Price, Phys. Rev. D 56, 6336 (1997).

[19] C. F. Sopuerta, N. Yunes, and P. Laguna, Phys. Rev. D p. 124010 (2006), astro-ph/0608600.

[20] C. F. Sopuerta, N. Yunes, and P. Laguna (2006), astro$\mathrm{ph} / 0611110$.

[21] P. Anninos and S. Brandt, Phys. Rev. Lett. 81, 508 (1998), gr-qc/9806031.
[22] M. Campanelli, Class. Quantum Grav. 22, S387 (2005), astro-ph/0411744.

[23] M. Campanelli, C. O. Lousto, P. Marronetti, and Y. Zlochower, Phys. Rev. Lett. 96, 111101 (2006), grqc/0511048.

[24] J. G. Baker, J. Centrella, D.-I. Choi, M. Koppitz, and J. van Meter, Phys. Rev. Lett. 96, 111102 (2006), grqc/0511103.

[25] F. Pretorius, Class. Quantum Grav. 22, 425 (2005), grqc/0407110.

[26] F. Herrmann, D. Shoemaker, and P. Laguna (2006), grqc/0601026.

[27] J. G. Baker et al., Astrophys. J (2007), astro-ph/0603204.

[28] J. A. González, U. Sperhake, B. Brügmann, M. Hannam, and S. Husa, Phys. Rev. Lett. (2006), gr-qc/0610154.

[29] F. Herrmann, I. Hinder, D. Shoemaker, P. Laguna, and R. Matzner (2007), gr-qc/0701143.

[30] M. Koppitz et al. (2007), gr-qc/0701163.

[31] D.-I. Choi et al. (2007), gr-qc/0702016.

[32] J. D. Schnittman and A. Buonanno (2007), astro$\mathrm{ph} / 0702641$.

[33] M. Campanelli, C. O. Lousto, Y. Zlochower, and D. Merritt (2007), version 1, http://www . arxiv.org/abs/gr-qc/0701164v1.

[34] B. Brügmann et al. (2006), gr-qc/0610128.

[35] U. Sperhake (2006), gr-qc/0606079.

[36] Cactus Computational Toolkit homepage, URL http://www. cactuscode.org/

[37] E. Schnetter, S. H. Hawley, and I. Hawke, Class. Quantum Grav. 21, 1465 (2004), gr-qc/0310042.

[38] M. Ansorg, B. Brügmann, and W. Tichy, Phys. Rev. D 70, 064011 (2004), gr-qc/0404056.

[39] J. E. McClintock et al., Astrophys. J (2007), astro$\mathrm{ph} / 0606076$.

[40] D. Merritt, M. Milosavljević, M. Favata, S. Hughes, and D. Holz, Astrophys. J 607, L9 (2004), astro-ph/0402057.

[41] M. Volonteri, F. Haardt, and P. Madau, Astrophys. J 582, 559 (2003), astro-ph/0207276.

[42] M. Volonteri and R. Perna, MNRAS 358, 913 (2005), astro-ph/0501345.

[43] M. Boylan-Kolchin, C.-P. Ma, and E. Quataert, Astrophys. J 613, L37 (2004), astro-ph/0407488.

[44] N. I. Libeskind, S. Cole, C. S. Frenk, and J. C. Helly, MNRAS 368, 1381 (2006), astro-ph/0512073.

[45] D. Merritt et al., MNRAS 367, 1746 (2006), astro$\mathrm{ph} / 0511315$.

[46] R. O'Leary, E. O'Shaughnessy, and F. Rasio (2007), astro-ph/0701887.

[47] Z. Haiman, Astrophys. J 613, 36 (2004), astro$\mathrm{ph} / 0404196$.

[48] P. Madau and E. Quataert, Astrophys. J. 606, L17 (2004).

[49] E. J. M. Colbert and R. F. Mushotzky, Astrophys. J 519, 89 (1999), gr-qc/9901023.

[50] F. Herrmann, I. Hinder, D. Shoemaker, and P. Laguna, Presented at "NRm3PN Meeting", St. Louis, February 8-11, 2007.

[51] J. G. Baker et al. (2007), astro-ph/0702390.

[52] M. Campanelli, C. O. Lousto, Y. Zlochower, and D. Merritt (2007), final version, http://www . arxiv.org/abs/gr-qc/0701164. 